\title{
Progress in the Development of the Standardized Public Health Emergency Preparedness Terminology
}

\author{
Nikolay Lipskiy ${ }^{1}$, James Tyson ${ }^{1},{ }^{2}$, Shauna Mettee Zarecki ${ }^{1}$, Jacqueline Burkholder ${ }^{1}$ \\ ${ }^{1}$ OPHPR, DEO, CDC, Atlanta, Georgia, United States, ${ }^{2}$ United States Public Health Service Commissioned Corps, Atlanta, Georgia, United States \\ Objective
}

The purpose of this project is to demonstrate the progress in development of a standardized public health (PH) emergency preparedness and response data ontology (terminology) through collaboration between the Centers for Disease Control and Prevention (CDC), Division of Emergency Operations (DEO), and the Logical Observation Identifiers Names and Codes (LOINC) system.

\section{Introduction}

The U.S. Department of Homeland Security National Incident Management System (NIMS) establishes a common framework and common terminology that allows diverse incident management and support organizations to work together across a wide variety of functions and hazard scenarios [1]. Using common terminology helps avoid confusion and enhances interoperability, particularly in fast-moving public health $(\mathrm{PH})$ emergency responses. In addition, common terminology allows diverse incident management and support organizations to work together across a wide variety of functions and scenarios [1]. LOINC is one of a suite of designated standards for the electronic exchange of public health and clinical information. Implementation of LOINC facilitates improvement of semantic interoperability, including unified terminology [2]. More than 68,100 registered users from 172 countries use LOINC to move interoperable data seamlessly between systems [3]. The CDC Division of Emergency Operations (DEO) leads development of standardized PH emergency preparedness and response terminology to improve effective and interoperable communications between national and international partners. Realizing the scale of LOINC support and implementation across the global public health arena, CDC DEO collaborates with LOINC to further enhance and harmonize the current PH emergency response terminology and to attain critical PH emergency management and preparedness and response requirements.

\section{Methods}

DEO analyzed 87,863 LOINC terms that were included in LOINC version 2.64, released on 06/15/2018 [3]. Access to this LOINC version was obtained through the Regenstrief LOINC Mapping Assistant (RELMA). RELMA is a Windows-based LOINC utility developed by the Regenstrief Institute (Indiapolis, USA) for searching the LOINC database and mapping local codes to LOINC codes [4]. The relevance of LOINC terminology to PH emergency preparedness and response was assessed through evaluating existing LOINC terminology against terminology specified by the World Health Organization PH Emergency Operation Centers (EOC). The following functions were evaluated: 1) Managing and Commanding; 2) Operating; 3) Planning/Intelligence; 4) Logistics and 5) Finance/Administration [5]. LOINC terminology was also evaluated against the CDC PH EOC Minimum Data Set (MDS) [6] that contains 315 standardized terms.

Analysis of fully specified LOINC terms was conducted through assessment of such LOINC term parts (attributes) as the code, name (component), system, method and class. Recommendations of gaps and enhancements were coordinated with LOINC management for inclusion of the new terminology in the release of version 2.65 .

\section{Results}

A new LOINC method, "CDC.EOC", is under development. Currently, the "emergency management incident" terminology presented by LOINC is limited by such characteristics as event type, event location and event name and requires amplification regarding to $\mathrm{PH}$ operations (i.e., communication, logistics etc.).

As a result of this investigation, emergency management terms are now being classified according to the type of incident or event (i.e., hurricane, outbreak, etc.) under LOINC code 80394-0. Similarly laboratory and clinical terms are being classified under a provisional LOINC code (89724-9). Two panels were created: 1) The emergency medical systems from the National Emergency

SDS Annual Conference Proceedings 2019. This is an Open Access article distributed under the terms of the Creative Commons AttributionNoncommercial 4.0 Unported License (http://creativecommons.org/licenses/by-nc/3.0/), permitting all non-commercial use, distribution, and reproduction in any medium, provided the original work is properly cited. 
Medical Services Information System (NEMSIS) was added under the NEMSIS.Panel $(\mathrm{n}=177$ terms) and 2) the Data Elements for Emergency Departments Systems (DEEDS) panel $(\mathrm{n}=152$ terms) was added with two subpanels: Attach.ED and Panel.ED

Assessing existing LOINC taxonomy and codification, DEO is working with the LOINC management team on evaluating additional options for reconciliation the $\mathrm{PH}$ emergency preparedness and response common information exchange reference model and LOINC standard. This process aims to further improve semantic interoperability of PH emergency preparedness and response information.

\section{Conclusions}

The LOINC terminology standardization is essential for improving PH preparedness and response data exchange and semantic interoperability. Collaboration with the Regenstrief Institute (LOINC) allows CDC to meet the terminology needs of PH emergency management and defines new opportunities for reconciliation data exchange between NIMS partners. This collaborative effort incorporates critically needed PH emergency and preparedness terminology and hierarchical structure in the LOINC standard.

\section{References}

1. FEMA National Incident Management System. Third Edition, October 2017. At: https://www.fema.gov/medialibrary-data/1508151197225- ced8c60378c3936adb92c1a3ee6f6564/FINAL_NIMS_2017.pdf

2. US National Library of Medicine. Logical Observation Identifiers Names and Codes (LOINC). At: https://www.nlm.nih.gov/research/umls/loinc_main.html

3. LOINC. The international standard for identifying health measurements, observations, and documents. At: https://loinc.org/

4. RELMA-the Reginstrief Institute LOINC Mapping Assistant. At: https://loinc.org/relma/

5. WHO. Framework for a Public Health Emergency Operations Centre. Interim document. November, 2015. At: http://www.who.int/ihr/publications/9789241565134_eng/en/

6. CDC. Public Health Information Network Vocabulary Access and Distribution System (PHIN VADS). Minimum Data Set for PH Emergency Operations Center. At: https://phinvads.cdc.gov/vads/SearchVocab.action 